\title{
Chest physiotherapy in newborns after surgical treatment of esophageal atresia: the alternative of ventilatory support in Madagascar
}

Keywords: chest physiotherapy, physical therapy, intensive care, newborn, esophageal atresia

\section{Introduction}

Because the thoracotomy is realized for surgical treatment of esophageal atresia (EA) with or without tracheal fistula (TF), postoperative respiratory complications are often observed. These complications may be poorly tolerated by neonates because of their age and their respiratory dynamics and airways physiological characteristics. Physiotherapy is regularly prescribed for optimizing airways efficiency; but very few studies demonstrate its effectiveness. ${ }^{1-3}$ The expected benefits are both prevention and treatment of respiratory complications, frequently observed after thoracic surgery. The aim of respiratory physiotherapy, from the newborn to the child is to limit or even to avoid bronchial congestion, which is a source of alveolar hypoventilation and atelectasis.

After surgical treatment of esophageal atresia with or without tracheal fistula, neonates are often kept under mechanical ventilation, at least for the first postoperative 24 hours. According to Patil et al., ${ }^{4}$ elective postoperative ventilation in the management of EA with TEF allows the protection of the esophageal anastomosis following repair of esophageal atresia with trachea-esophageal fistula. Using ventilatory support in early post-operative period avoids the need for initial vigorous physiotherapy. Thus, the anastomosis can be rested and protected from injury; moreover, the prevention of postoperative respiratory distress could maintain adequate tissue $\mathrm{PaO}_{2}$.

Furthermore, the waking period is vital in the newborn after general anesthesia. Indeed, the residual effect of the anesthetic agents leads to depression of the respiratory centers associated with alveolar hypoventilation, a decrease in lung compliance and functional residual capacity, and atelectasis. Then, respiratory automatism is inhibited, as are swallowing and coughing reflexes. This involves postoperative mechanical ventilation until the complete recovery of the patient's respiratory autonomy, ${ }^{5}$ as well as physiotherapy to prevent or even to avoid atelectasis (Figure 1A \& 1B).

In our daily practice, in Madagascar, respiratory physiotherapy has been prescribed early to prevent the consequences of bronchial and alveolar obstruction, to reduce the consequences of per operative mechanical ventilation, to reduce respiratory work by techniques focused on pulmonary recruitment. ${ }^{5}$ After surgical treatment of esophageal fistula, session of respiratory physiotherapy began from the first postoperative day. The duration and the intensity of the physiotherapy sessions were adapted to the postoperative health status of the child (weariness, pain).

From January 1st, 2017 to June 30th, 2017, we recorded five cases of esophageal atresia with tracheal fistula. For most of those newborns, hospital admission was at one-day of life, motivated by respiratory distress after breastfeeding. Surgery was performed under general
Volume I Issue 4 - 2017

\author{
Harifetra Mamy Richard Randriamizao,' \\ Aurelia Rakotondrainibe,' Mamy Lalatiana \\ Andriamanarivo ${ }^{2}$ \\ 'Department of Anesthesiology and Resuscitation, University of \\ Antananarivo, Madagascar \\ ${ }^{2}$ Department of Surgery, University of Antananarivo, Madagascar
}

\begin{abstract}
Correspondence: Rakotondrainibe Aurélia, Faculty of Medicine, Department of Anesthesiology and Resuscitation, University of Antananarivo, Surgical Intensive Care Unit - $\mathrm{CHU}$ HJRA, BP 4I50 Antananarivo I0I, Madagascar, Tel +26I 3440 326 22, Email aaurelia.rakotondrainibe@gmail.com
\end{abstract}

Received: September 07, 2017 | Published: December 18, 2017

anesthesia with tracheal intubation. The lack of adequate equipment for mechanical ventilation (even in the very short term) in newborns makes us advocate of a gradual awakening with assisted ventilation. This assisted ventilation gradually moves towards spontaneous ventilation. Practically, a session of respiratory physiotherapy is daily realized for EA operated neonate. The administration of bronchodilator by aerosol and optimization of analgesia (with morphine derivatives (nalbuphine)) proceeded each physiotherapy session.

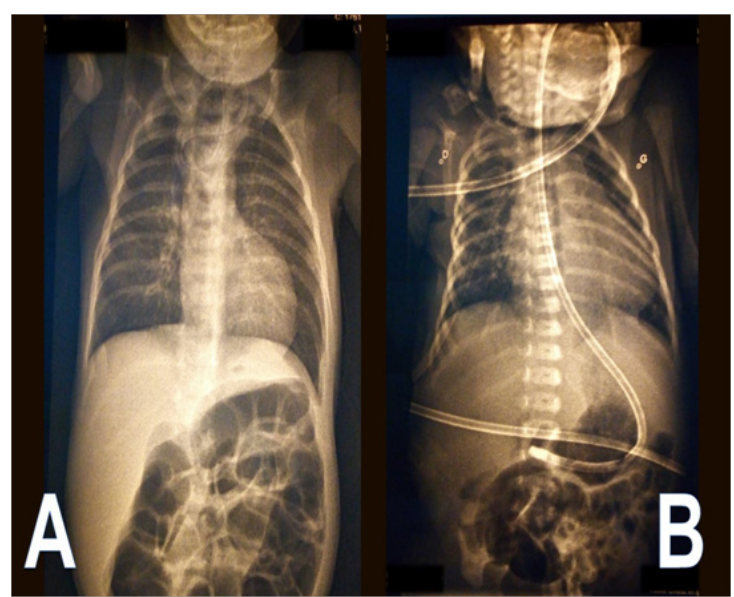

Figure I (A) Preoperative chest X-ray showing the progression of the gastric tube in the proximal esophageal pouch. (B) Postoperative chest X-ray, realized on the fourth day after the surgical management of EA, showing the absence of pulmonary atelectasis, after a daily session of respiratory physiotherapy.

Gentle physiotherapy maneuvers, allow to evacuate the bronchial and alveolar mucous plug. The technique used is mainly the technique of expiratory flow acceleration, which is based on the principle of mobilization of the pulmonary secretions, from the distal to the proximal. ${ }^{6}$ This technique is performed by a chest pressure and an 
abdominal abutment. During expiration, the practitioner applies pressure to the rib cage of the neonate. These maneuvers are usually carried out in supine position and lateral decubitus in order to be elective on the treated pulmonary territory; then after, a pharyngeal aspiration of the secretions is required at the end of each physiotherapy session. This daily session of physiotherapy lasted an average of five postoperative days, until improvement of the breathing function of the newborn.

\section{Conclusion}

The benefits and potential risks of physiotherapy must be well evaluated. Its widespread use unfortunately relies more on personal habits and beliefs than on scientific evidence. Physiotherapy also remains an "art" but the main inconvenient is the personal variable of the practitioner. ${ }^{2}$ Despite the physiotherapy techniques that we use with the seemingly positive prognosis (both in the short and medium term), caution remains as other studies are not available.

\section{Acknowledgements}

None.

\section{Conflict of interest}

The author declares no conflict of interest.

\section{References}

1. Wallis C, Prasad A. Who needs chest physiotherapy? Moving from anecdote to evidence. Arch Dis Child. 1990;80(4):393-397.

2. Stiller K. Physiotherapy in intensive care: towards an evidence-based practice. Chest. 2000;118(6):1801-1813.

3. Jelic S, Cunningham JA, Factor P. Clinical review: airway hygiene in the intensive care unit. Crit Care. 2008;12(2):209.

4. Patil RT, Prakash A. Strategies for optimizing surgical outcome in patients with esophageal atresia with tracheo-esophageal fistula. Int Surg J. 2017;4(5):1706-1709.

5. Demont B. La kinésithérapie respiratoire en service de réanimation néonatale, mise au point. Kiné Réa. 2007;47:13-17.

6. Guyet D, Guillier A, Carme T, et al. Kinésithérapie et désencombrement des voies aériennes inférieures par stimulations de la toux réflexe et/ou du réflexe expiratoire chez le nouveau-né et le nourrisson. Kinésithér Scient. 2005;568:13-23. 